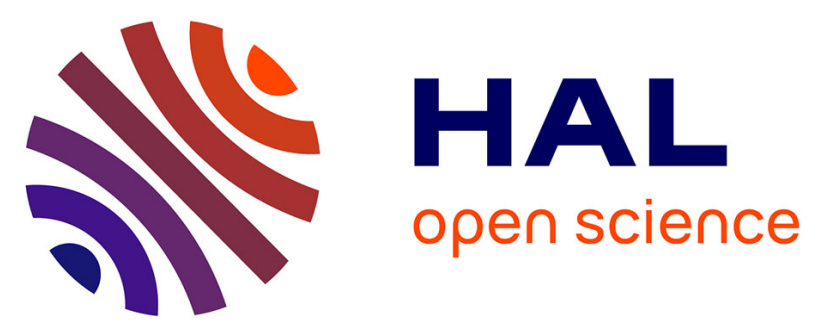

\title{
Usage of characteristic modes with specific boundary conditions for wide-band antenna design: application to a cage loaded monopole antenna
}

Bernard Souny, Christophe Morlaas

\section{- To cite this version:}

Bernard Souny, Christophe Morlaas. Usage of characteristic modes with specific boundary conditions for wide-band antenna design: application to a cage loaded monopole antenna. IEEE Transactions on Antennas and Propagation, 2018, 66 (8), pp. 3819 - 3829. 10.1109/TAP.2018.2835516 . hal01799022

\section{HAL Id: hal-01799022 \\ https://hal-enac.archives-ouvertes.fr/hal-01799022}

Submitted on 24 May 2018

HAL is a multi-disciplinary open access archive for the deposit and dissemination of scientific research documents, whether they are published or not. The documents may come from teaching and research institutions in France or abroad, or from public or private research centers.
L'archive ouverte pluridisciplinaire HAL, est destinée au dépôt et à la diffusion de documents scientifiques de niveau recherche, publiés ou non, émanant des établissements d'enseignement et de recherche français ou étrangers, des laboratoires publics ou privés. 


\title{
Usage of characteristic modes with specific boundary conditions for wide-band antenna design: application to a cage loaded monopole antenna.
}

\author{
Bernard Souny, Christophe Morlaas
}

\begin{abstract}
In this article, a technique to design a compact wideband antenna with the optimization of the input impedance is proposed. This technique is based on the use of two sets of characteristic modes. The first one is associated to an open circuit at the antenna input port while the second is associated to a short circuit at the antenna input port. The link of this analyze with a classical modal approach by a rational fraction approximation of the input impedance is demonstrated. This technique is firstly applied to a basic folded monopole antenna, then to a disc-loaded folded monopole antenna in the aim to optimize its bandwidth and radiation pattern while reducing its size. The disc-loaded folded monopole antenna is modeled and measured. Its VSWR performances are equivalent to thus of the Goubau antenna for equivalent dimensions. The radiation pattern is monopole-like on the full bandwidth.
\end{abstract}

Index Terms - compact antenna, wide bandwidth, characteristic modes, modal analysis, poles and zeros, rational fraction Goubau antenna, loaded antenna, monopole antenna.

\section{INTRODUCTION}

W ITH the development of the wireless telecommunications, the use of wide bandwidth systems become an obligation. In many applications it is important to minimize the antenna size, which is however constraint by the physical laws [1].

The operating frequency band of the antenna is essentially linked to four notions: the maximal voltage standing wave ratio (VSWR) in the frequency band that is often fixed at 2, the radiation pattern, the polarization purity, and the efficiency.

In many applications, monopole-like pattern is required and compact monopole-like structures with wide-band properties are still extensively studied [2], [3]. A kind of antenna that can be used is the disk loaded folded monopole and a well known type of such a wide bandwidth miniaturized monopole is the antenna proposed by G. Goubau [4] that presents one octave bandwidth in term of VWSR with a $\lambda / 10$ height and a $\lambda / 10$ radius at the lower frequency.

For small antennas the maximum usable bandwidth is frequently defined by the Chu limit [5], which links the quality factor to the antenna dimension. However, this quality factor is only linked to the bandwidth if the input reflection coefficient of the antenna only depends on one pole close to the useful frequency band [6].

C. Morlaas is with ENAC, TELECOM-EMA, Toulouse, F-31055 France e-mail: morlaas@recherche.enac.fr. He is also affiliated with Université de Toulouse, F-31400 Toulouse, France.

B. Souny is an associate researcher with ENAC, TELECOM-EMA.
The poles of the reflection coefficient correspond to the eigenmodes of the structure loaded with a matched waveguide at the input. Generally they are complex. Some pure imaginary poles can be used to improve the matching [7], [8].

Additional poles that contribute to the radiation can also be used [9], [10] to improve the bandwidth. However the radiation pattern and the polarization quality can be strongly affected by their radiation.

Characteristic modes have been initially introduced by Garbacz and Harrington [11], [12], [13], [14]. More recently, the mode characteristics have been used to facilitate the antenna design [15], [16], [17], [18]. In this article, we propose to link the characteristic mode to an poles analysis of an antenna input impedance or admittance also known as the modal analysis [19]. An approximation by fractional ration is used in which, an open-circuit (OC) and a short-circuit (SC) at the input of the antenna are considered. Similarly, This analysis can be performed with the zeros of the impedance and the admittance. The link between these poles and the characteristic modes of the antenna in the two input configurations as mentioned above, i.e. OC and SC, is shown. We show how this analysis can be useful to optimize the input impedance bandwidth and the radiation of a wide-band antenna. As an example, the proposed method is applied to a simple folded monopole antenna and to a more complex case of a folded monopole loalded by a metallic disc already published in [20] which constitute an upgrade of the Goubau antenna [4].

The section II presents the relations between classical modes analysis and characteristic modes of Garbacz/Harrington. Then, the usefulness of consider two input boundary conditions, i.e. an open circuit and a short circuit, at the input of a wide-band antenna is explained and illustrated for a folded monopole antenna. From this analysis the important points that affect the antenna design are reported and a new design is proposed.

The section III describes the classical mode and characteristic mode analysis of the improved antenna structure. The performances of this antenna as well as the ones of an optimized antenna structure are presented.

Finally, in section IV, the measurement performances of the optimized antenna version is presented.

The simulations has been done with FEKO and the rational fraction approximation is computed with OCTAVE. 


\section{RELATIONS BETWEEN CLASSICAL MODES ANALYSIS} AND CHARACTERISTIC MODES OF GARBACZ/HARRINGTON

In this part, the link between a classical modal analysis and characteristic modes is shown. The usefulness of characteristic modes analysis with short-circuited or open-circuited boundary conditions according to the kind of antenna is depicted.

\section{A. Modal analysis}

The modal analysis based on the zeros and poles representation of the input impedance by a rational fraction is a classical technique for antennas study [19]. The eigenmodes are generally associated to a complex pulsation due to the radiation phenomena. Poles of the impedance are linked to eigenmodes of the antenna structure with an open-circuited input and zeros of the impedance are linked to eigenmodes of the antenna with a short-circuited input and vice-versa for the admittance. Thus, the frequency behavior of the antenna can only be described in term of modes.

Theoretically each set of modes with SC and OC input conditions allows to describe the antenna performances through its input port, but as it will be seen, it is more useful to consider the two sets of modes together.

This analysis is suitable for the study of small antenna because only few poles and zeros takes action into the all bandwidth. indeed, complex poles and zeros of the input impedance are defined by pair of conjugate numbers but only the ones around the useful bandwidth are retained.

The process to obtain the poles and zeros is described as follows.

1) The input reflection coefficient of an antenna is measured or computed.

2) The input impedance is computed from the reflection coefficient $(\Gamma)$ with $z_{i n}=(1+\Gamma) /(1-\Gamma)$ and poles of impedance are computed by a classical method [21] [22] [23] [24].

3) The input admittance is computed from the reflection coefficient $(\Gamma)$ with $y_{i n}=(1-\Gamma) /(1+\Gamma)$ and poles of admittance that are zeros of impedance are computed by the same classical method.

Let us take the case of a dipole antenna, The reflection coefficient obtained with a simulation between $400 \mathrm{MHz}$ and 2 $\mathrm{GHz}$ and the rational fraction results are plotted in the complex plane in Fig. 1.

The rational fraction gives a good approximation of the simulated points with the numerator and denominator order equal to 3. The frequency and quality factor of useful poles and zeros close to the analysis band are given in (1)

$$
\begin{array}{llrl}
f z_{1} & =0.894 \mathrm{GHz}, & & Q z_{1}=5.3 \\
f p_{1} & =1.74 \mathrm{GHz}, & & Q p_{1}=3
\end{array}
$$

with $Q$ defined in appendix VI

\section{B. Influence of the kind of excitation on characteristic modes}

The electrical and magnetic impress currents $J_{s}, M_{s}$ defined in the Maxwell equations [25] which contribute to the incident field [12] are introduced in the characteristic modes theory

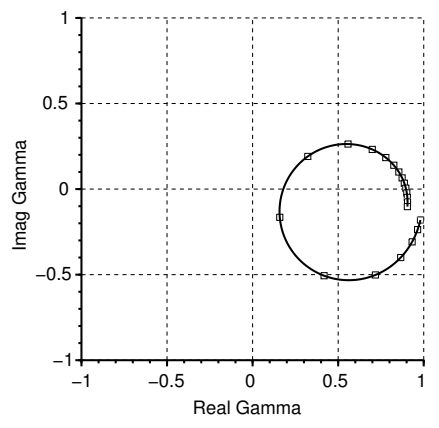

Fig. 1. Real versus imaginary part of the input reflection coefficient of the dipole antenna between $400 \mathrm{MHz}$ and $2 \mathrm{GHz}$. The square points correspond to the simulation values. The full line corresponds to the interpolation with the rational fraction.

without difficulties. It is well established that the characteristic modes associated to a fixed structure defined by materials and boundary conditions do not depend on the sources $J_{s}$ and $M_{s}$. Then, the modification of their amplitude and position does not change characteristic modes. Besides, the excitation of an antenna implies the definition of a port associated to a boundary condition before to apply the source. Well, characteristic modes depend on boundary conditions and altough a superposition of characteristic modes always allow to obtain the excited fields whatever the source because they defined a complete base, the choice of the appropriate boundary conditions allow to use the minimum number of characteristic modes to describe the excited field.

In theoretical work current or voltage sources are very often used [25]. An example of sources compliant with a dipole antenna is presented in Fig. 2. These sources are connected to the antenna by the areas $\Sigma 1$ and $\Sigma 2$.

Boundary conditions associated with these sources are a short circuit (Perfect electrical conductor) for a voltage source (Fig. 2-a) and an open circuit (Perfect magnetic conductor) for a current source (Fig. 2-b). In practice this latter is often approached by no conductor, obtained by neglecting the displacement currents (Fig. 2-c).

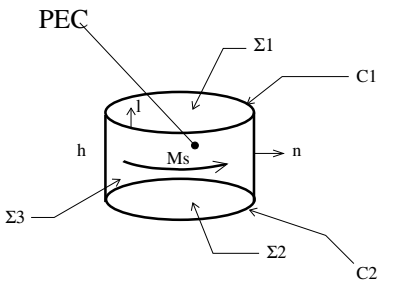

(a) Voltage source with PEC

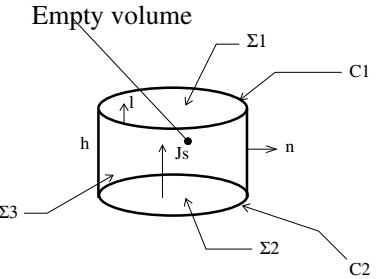

(c) Simplified current source
Fig. 2. Classical lumped sources 
But in practice antennas are generally fed through a port on which a matched source with a finite internal resistance is put,

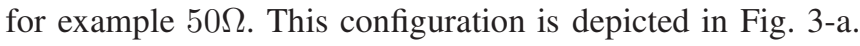

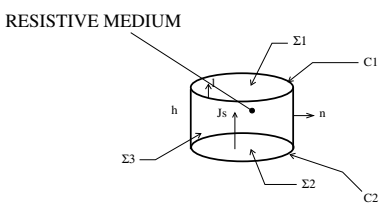

(a) Resistive source

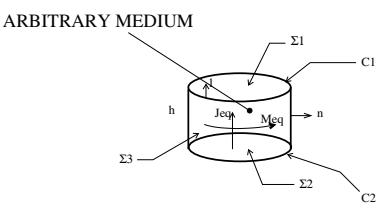

(b) Equivalent resistive source
Fig. 3. Equivalent real source.

In this case, the use of the equivalent theorem allows to replace the real source by equivalent (impress) sources $J_{e q}$ and $M_{e q}$ with the internal media replaced by an arbitrary media as shown in Fig. 3-b. If the media is replaced by a perfect electrical conductor (PEC), that corresponds to a voltage source. If the media is replaced by a perfect magnetic conductor (PMC), that corresponds to a current source.

Fig. 4 shows the $\lambda / 2$ dipole with a feeding port at its center using sources defined in Fig.2. Fig. 4-a corresponds to a voltage source while 4-b corresponds to an approximate current source.

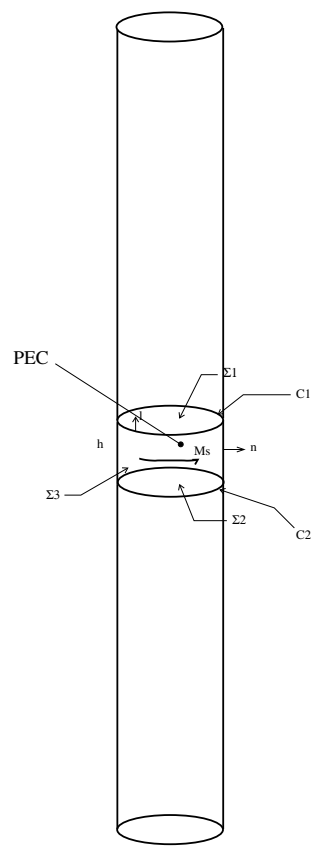

(a) Dipole with voltage source

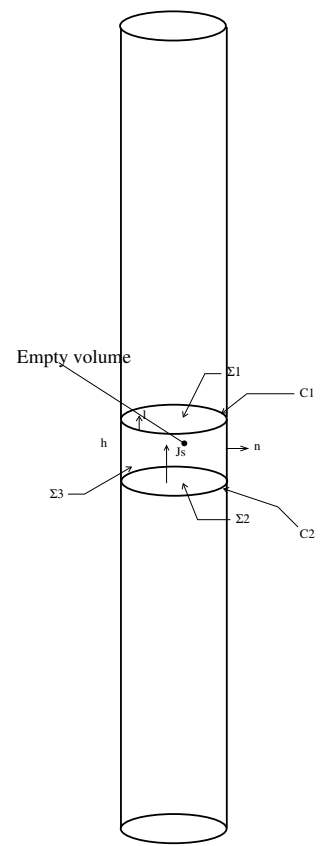

(b) Dipole with current source
Fig. 4. Half-wave dipole with voltage and current excitation.

We observe that the excitation by mean of a voltage source corresponds to the half-wavelength dipole fed with a loop of a magnetic current located at its center but the excitation by mean of a current source corresponds to a structure completely different due to the difference of the boundary conditions: two dipole of half length.

For instance, consider a dipole of $l=150 \mathrm{~mm}$ length and a $d=2 \mathrm{~mm}$ diameter. Frequencies obtained with a characteristic mode analysis with an OC input (poles of impedance) and a $\mathrm{SC}$ input (zeros of impedance) are given in (2).

$$
\begin{aligned}
& f c z_{1}=0.935 \mathrm{GHz} \\
& f c p_{1}=1.830 \mathrm{GHz} \\
& f c z_{2}=1.927 \mathrm{GHz}
\end{aligned}
$$

We observed that frequencies extracted from the rational fraction approximation given in (1) are close to the ones of the characteristic modes analysis given in (2). Nevertheless, a slight difference exists because the associated quality factors have low values.

The characteristic modes for the two source configurations (SC/OC) are very different as it can be seen in Fig. 5. The currents of the first characteristic mode with a SC input as boundary condition correspond to the ones of the fundamental mode of a $l=150 \mathrm{~mm}$ length dipole (Fig. 5-a). The currents of the first characteristic mode with an OC input as boundary condition correspond to the ones of the fundamental mode of two dipoles of $l=75 \mathrm{~mm}$ length with a odd symmetry (Fig. 5b). While the currents of the second characteristic mode with an SC input as boundary condition correspond to the ones of the fundamental mode of two dipoles of $l=75 \mathrm{~mm}$ length with an even symmetry (Fig. 5-c).

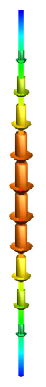

(a) First characteristic mode with SC input at $935 \mathrm{MHz}$

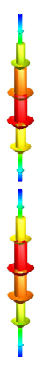

(b) First characteristic mode with OC input at $1830 \mathrm{MHz}$

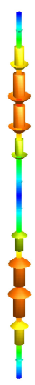

(c) Second characteristic mode with SC input at $1927 \mathrm{MHz}$

Fig. 5. First characteristic modes with a short-circuited input and an opencircuited input for the half-wave dipole.

In the appendix VI, the link between eigenmodes and characteristic modes with OC and SC input for isolated modes is demonstrated. The relevance of the isolation of a mode for an isomorphic operation associated to a pole $p_{n}=\alpha_{n}+j \omega_{n}$ depends on the proximity of the others modes and of the associated quality factor.

In the following we assume that modes can be considered as isolated. Therefore, when the operating frequency is close to the one of an isolated characteristic mode with a SC input the actually used source can be replaced by an equivalent voltage 
source without any fields modification. The characteristic modes are then the ones of the structure with a SC input as boundary condition and the isolated state of the mode close to the operating frequency induces that the excited field is essentially the one of the considered characteristic mode.

Dualistically, when the operating frequency is close to the one of an isolated characteristic mode with an OC input the actually used source can be replaced by an equivalent current source without any fields modification. The characteristic modes are then the ones of the structure with an OC input as boundary condition and the isolated state of the mode close to the operating frequency induces that the excited field is essentially the one of the considered characteristic mode.

In practice, to observe the currents on the antenna structure, an impedance matching source is used.

The currents of the dipole excited by a resistive source are given in the Fig. 6-a, b, c at the frequency $f c z_{1}, f c p_{1}$ and $f c z_{2}$ respectively. At $f c z_{1}$, the currents are very similar to the ones of the characteristic mode with SC as boundary condition at this frequency (Fig. 5-a). At $f c p_{1}$ and $f c z_{2}$, the currents are very similar to the ones of the characteristic mode with OC as boundary condition at $f c p_{1}$ (Fig. 5-b). The mode corresponding to the characteristic mode at $f c z_{2}$ is not excited because it presents an even symmetry and cannot be excited by a source at the center of the dipole.

Because characteristic modes constitute a complete base, they always allow to represent the excited field and close to $f c p 1$ we can represent fields as a superposition of characteristic modes with SC boundary condition but it will be far simpler to represent it with only one characteristic mode with OC boundary. So it will be very easy to predict the dipole operation close to $f c p 1$ using only this characteristic mode.

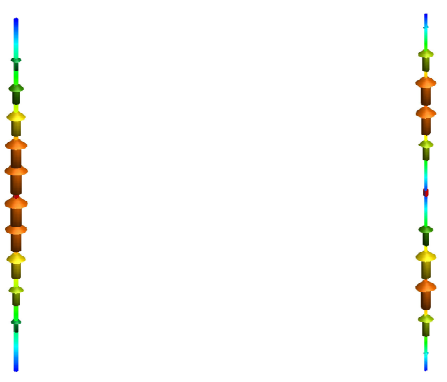

(a) Half-wave dipole exited at (b) Half-wave dipole exited at $935 \mathrm{MHz}\left(f c z_{1}\right)$ $1830 \mathrm{MHz}\left(f c p_{1}\right)$

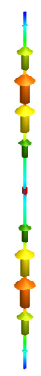

(c) Half-wave dipole exited at $1927 \mathrm{MHz}\left(f c z_{2}\right)$

Fig. 6. Currents of the half-Wave dipole exited with a resistive source.

For narrow operating bandwidth, two kinds of antennas

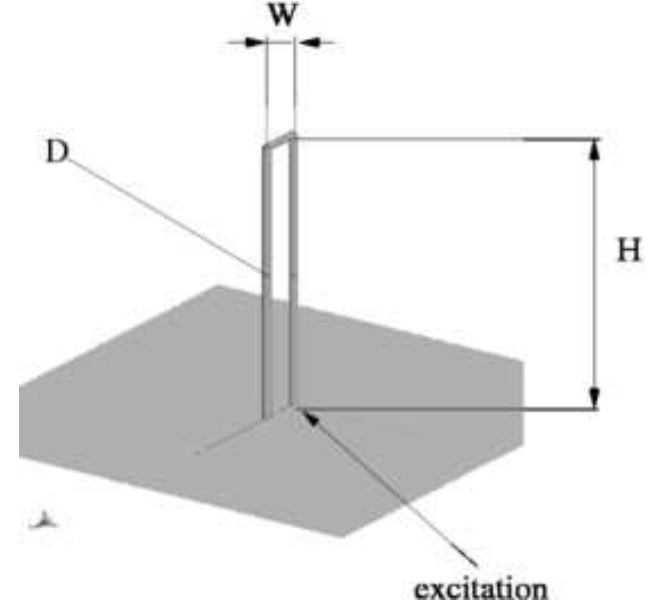

Fig. 7. Basic folded-monopole. $\mathrm{H}=74 \mathrm{~mm}, \mathrm{~W}=10 \mathrm{~mm}, \mathrm{D}=2 \mathrm{~mm}$.

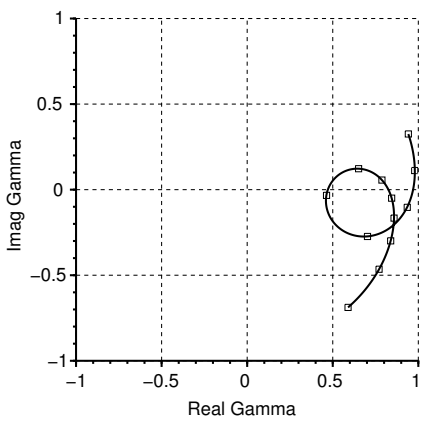

Fig. 8. Real versus imaginary part of the input reflection coefficient of the basic folded monopole antenna between $400 \mathrm{MHz}$ and $1.7 \mathrm{GHz}$. The square points correspond to the simulation values. The full line corresponds to the interpolation with the rational fraction.

(resonant antennas) can be distinguished. The ones defined by only one pole of the impedance (a half-wavelength slot antenna for instance) for which the corresponding characteristic modes with OC boundary condition help the antenna analysis along. The ones defined by only one pole of the admittance (a half-wavelength dipole antenna for instance) for which the characteristic modes with SC boundary condition help the antenna analysis along.

Concerning the wide-band antennas, for which poles and zeros of the impedance act together, the characteristic modes analysis with SC and OC boundary conditions help the analysis along as shown in the following study of the folded monople and the suggested antenna.

\section{Illustration on a basic folded-monopole}

The analysis of a basic folded-monopole (Fig. 7) is given first because the modes of the proposed antenna are an evolution of the basic folded-monopole modes.

The reflection coefficient obtained with a simulation between $400 \mathrm{MHz}$ and $1.7 \mathrm{GHz}$ and the rational fraction results are plotted in the complex plane in Fig. 8.

The rational fraction gives a good approximation of the simulated points with the numerator and denominator order 


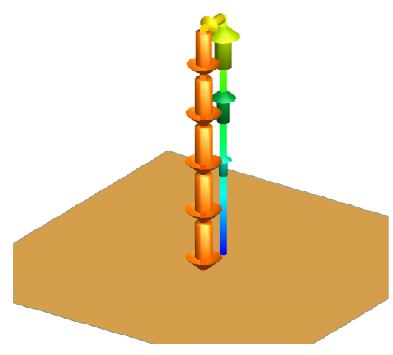

(a) Charact. mode OC input $0.562 \mathrm{GHz}$

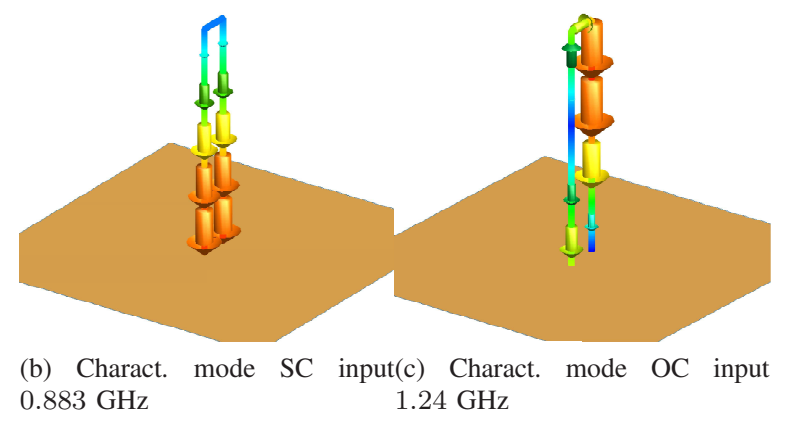

Fig. 9. Characteristic mode currents for a basic folded monopole antenna.

equal to 3 . The simulated point number used to build the approximation is not critical but they must be well spread over the curve.

The frequency and quality factor of useful poles and zeros close to the analysis band are given in (3)

$$
\begin{array}{ll}
f p_{1}=0.58 \mathrm{GHz}, & Q p_{1}=18.3 \\
f z_{1}=0.847 \mathrm{GHz}, & Q z_{1}=4.0 \\
f p_{2}=1.2 \mathrm{GHz}, & Q p_{2}=4.3
\end{array}
$$

Besides, frequencies obtained with a characteristic mode analysis with an open-circuited input (poles) and a shortcircuited input (zeros) are given in (4).

$$
\begin{aligned}
& f c p_{1}=0.562 \mathrm{GHz} \\
& f c z_{1}=0.883 \mathrm{GHz} \\
& f c p_{2}=1.24 \mathrm{GHz}
\end{aligned}
$$

Wire currents obtained with a characteristic mode analysis are plotted in Fig.9,

The characteristic mode at $f c p 1=0.562 \mathrm{GHz}$ (Fig. 9-a) corresponds to the $\lambda / 4$ resonant mode of the loop with an OC input. The characteristic mode at $f c p 2=1.24 \mathrm{GHz}$ (Fig. 9-c) corresponds to the $3 \lambda / 4$ resonant mode of the loop with an OC input and the characteristic mode at $f c z 1=0.883 \mathrm{GHz}$ (Fig. 9-b) corresponds to the $\lambda / 2$ resonant mode of the loop with a SC input which is the $\lambda / 4$ mode of the monopole antenna.

\section{Evolution of the antenna}

From some simulations it is easy to observe that $f c z 1$ mode have few dependence with the distance between the two vertical wires contrary to the modes at $f c p 1$ and $f c p 2$. After having identified the mode at $f c z 1=0.883 \mathrm{GHz}$,

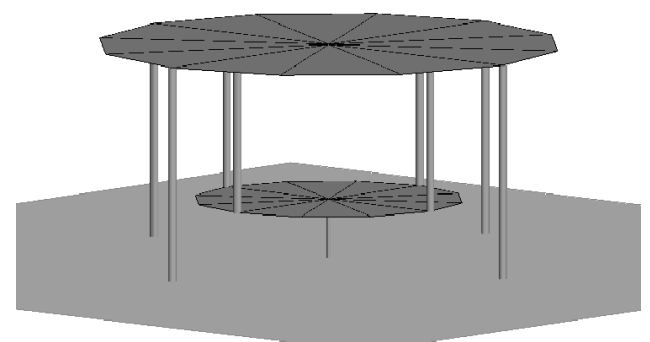

Fig. 10. Evolution $\mathrm{N}^{\circ} 1$ of the folded monopole.

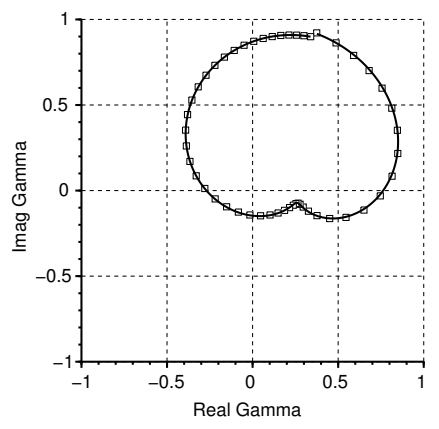

Fig. 11. Real versus imaginary part of the input reflection coefficient (Gamma) of the evolution $\mathrm{N}^{\circ} 1$ between $300 \mathrm{MHz}$ and $1.5 \mathrm{GHz}$. The square points correspond to the simulation values. The full line corresponds to the interpolation with the rational fraction.

this frequency can be reduced by increasing the size of the upper conductor by applying a top disc for example. This top disc allows also to reduce $f c p 1$ and $f c p 2$ as maintaining the bandwidth. Because the currents associated to the $f c p 1$ mode are strong onto the wire without the port (outer wire), this frequency can be decreased relatively to the one of the $f c p 2$ mode by lengthen this wire with a meander shape for example. Besides, the input impedance can be reduced by increasing the diameter of the wire containing the port.

Then, from this first analysis on the folded monopole, it is easy to optimize the distance between these wires to maximize the bandwidth and to reduce the antenna high. The first evolution of the folded monopole antenna is shown in Fig. 10 where the central conductor is a stepped design to allow to increase the diameter of the wire containing the port, to reduce the input impedance, and to simply connect it to a standard coaxial connector. To close the loop between the top disc to the ground plane four outer conductors are used to avoid an undulation of the radiation pattern at high frequency as explained in [20].

The input reflection coefficient is given in Fig. 11. This is typically a two poles and one zero inserted system.

Frequencies obtained with a modal analysis (left side) and a characteristic mode analysis (right side) with an open-circuited input (poles) and a short-circuited input (zeros) are given in 
(5).

$$
\begin{array}{ll}
f p_{1}=0.50 \mathrm{GHz} & f c p_{1}=0.50 \mathrm{GHz} \\
f z_{1}=.60 \mathrm{GHz} & f c z_{1}=.68 \mathrm{GHz} \\
f p_{2}=.775 \mathrm{GHz} & f c p_{2}=1.20 \mathrm{GHz} \\
f z_{2}=1.06 \mathrm{GHz} & f c z_{2}=1.375 \mathrm{GHz}
\end{array}
$$

$f c p_{1}$ and $f c z_{1}$ are too close to $f c p_{2}$ to obtain a wideband operation. Then, to decrease $f c p_{1}$ and $f c z_{1}$ a solution consists of lengthen the outer wires. To lengthen the wires different solutions can be used; a portion of helix, a meander line section, a solenoid section.

The simplest is the first one and it is the solution used in Fig. 12.

\section{MONOPOLE ANTENNA LOADED WITH A QUADRIFILAR CAGE}

\section{A. Antenna description}

This antenna is an evolution of the basic folded monopole, it is a cage loaded monopole antenna and is called the cage antenna.

A picture of this antenna is given in Fig. 12. The antenna is intended to be used on a metallic roof. Thus, for simulation purpose the antenna is over an infinite perfect electrical (IPEC) conductor. It consists of two circular discs linked each-other with four inner vertical wires (IW) of same diameters $(2 \mathrm{~mm})$. The bottom disc (BD) of $D 2=7 \mathrm{~cm}$ diameter is linked to the IPEC by mean of the coaxial feed wire (FW) and the top metallic disc (TD) of $D 1=12 \mathrm{~cm}$ diameter is linked to the IPEC via four outer helical shape wires (OW). The total height is $h 1=5.5 \mathrm{~cm}$.

During the design process, an idealized model is used to accelerate simulation: perfect conductor discs are infinitely thin.

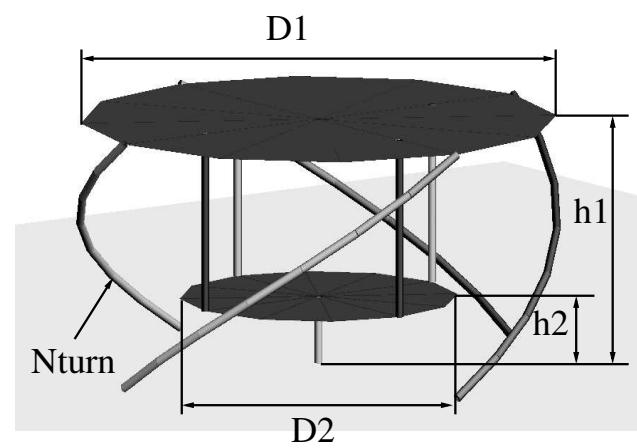

Fig. 12. Monopole antenna with quadrifilar cage (section III): $h 1=5.5$ $\mathrm{cm}, h 2=1.5 \mathrm{~cm}, D 1=12 \mathrm{~cm}, D 2=7 \mathrm{~cm}$, Wire diameter $2 \mathrm{~mm}$, Nturn $=.25$.

\section{B. Modal analysis}

The antenna input reflection coefficient (Gamma) is computed via a simulation between $300 \mathrm{MHz}$ and $1.8 \mathrm{GHz}$. It is plotted in Fig. 13.

The obtained rational fraction gives a good approximation of poles and zeros close to this frequency band with the

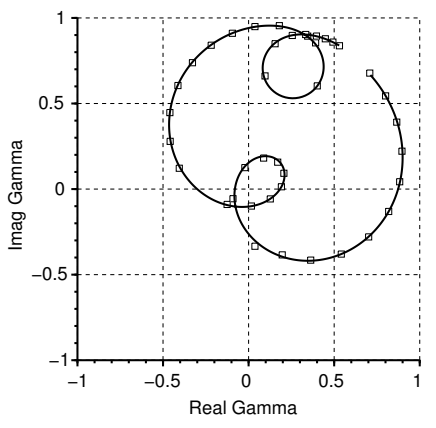

Fig. 13. Real versus imaginary part of the input reflection coefficient (Gamma) of the cage antenna between $300 \mathrm{MHz}$ and $1.8 \mathrm{GHz}$. The square points correspond to the simulation values. The full line corresponds to the interpolation with the rational fraction.

numerator and denominator order equal to 4 . The frequencies and quality factor of useful poles and zeros close to the analysis band are given in (6) with corresponding frequencies of associated characteristic modes. The analysis is extended to frequencies higher than the useful band to explain certain pattern deformation at high frequency.

$$
\begin{aligned}
& \left.f p_{1}=0.377 \mathrm{GHz}, \quad Q p_{1}=15.5 \quad f c p_{1}=0.386 \mathrm{GH} \nless 6\right) \\
& f z_{1}=0.539 \mathrm{GHz}, \quad Q z_{1}=3.2 \quad f c z_{1}=0.581 \mathrm{GHz} \\
& f p_{2}=0.806 \mathrm{GHz}, \quad Q p_{2}=2.1 \quad f c p_{2}=1.01 \mathrm{GHz} \\
& f z_{2}=1.126 \mathrm{GHz}, \quad Q z_{2}=5.5 \quad f c z_{2}=1.272 \mathrm{GHz} \\
& f p_{3}=1.55 \mathrm{GHz}, \quad Q p_{3}=35 \quad f c p_{3}=1.554 \mathrm{GHz}
\end{aligned}
$$

1) Mode $p_{1}: 377 \mathrm{MHz}$ : The antenna is excited at $377 \mathrm{MHz}$, the wire currents are plotted in part (a) of Fig. 14 where only one snapshot is given at phases $90^{\circ}$ because all pieces of current are in phase and maximal at this phase. Currents on the disc are essentially the continuation of the wire current and for readability, they are not plotted. We can see that the currents along the helical cage (OW) are strong while they are weak along the inner vertical wires (IW). In fact this is an evolution of the $p_{1}$ mode identified in the basic folded monopole (unfed wire). This mode is strongly dependent to the helical cage parameters: the length of the wires (this parameter depends on the turn number $N_{\text {turn }}$ ) and the upper disc (TD) diameter. The corresponding radiating mode is principally a $T M_{01}$.

The corresponding characteristic mode with an opencircuited input is find at $387 \mathrm{MHz}$. These currents are given in Fig. 14 (b). We can see that they are very similar to those obtained with the rational fraction process given in Fig. 14 (a).

2) Mode $z_{1}: 539 \mathrm{MHz}$ : The antenna is excited at $539 \mathrm{MHz}$, the wire currents are plotted in part (a) and (b) of Fig. 15 where two snapshots are given at phases $0^{\circ}$ and $90^{\circ}$ because all pieces of current are not in phase. The corresponding radiating mode is principally a $T M_{01}$.

The corresponding characteristic mode with a shortcircuited input is find at $581 \mathrm{MHz}$. These currents are given in Fig. 15 (c). We can see that they are similar but not identical to wire currents in antenna excited at $539 \mathrm{MHz}$. However we 

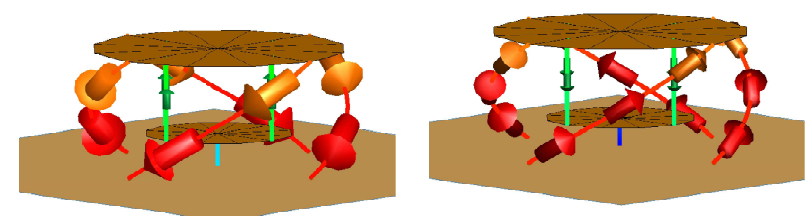

(a) $p_{1}$ mode $0.377 \mathrm{GHz}$, phase $90^{\circ}$

(b) Charact. mode $0.387 \mathrm{GHz}$

Fig. 14. $p_{1}$ mode and characteristic mode currents with OC input for the cage antenna.

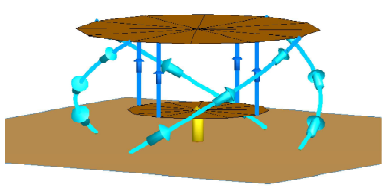

(a) $z_{1}$ mode $0.539 \mathrm{GHz}$, phase $0^{\circ}$

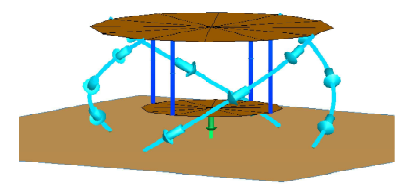

(b) $z_{1}$ mode $0.539 \mathrm{GHz}$, phase $90^{\circ}$

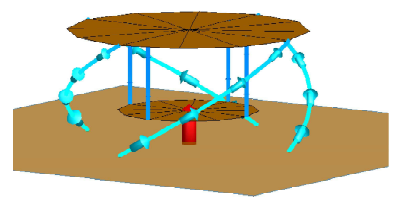

(c) Charact. mode $0.581 \mathrm{GHz}$

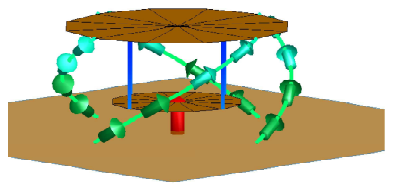

(a) $p_{2}$ mode $0.806 \mathrm{GHz}$, phase $0^{\circ}$

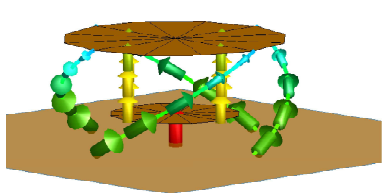

(b) $p_{2}$ mode $0.806 \mathrm{GHz}$, phase $90^{\circ}$

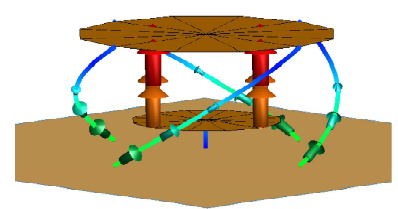

(c) Charact. mode $1.014 \mathrm{GHz}$

Fig. 16. $p_{2}$ mode and characteristic mode currents with OC input for the cage antenna.
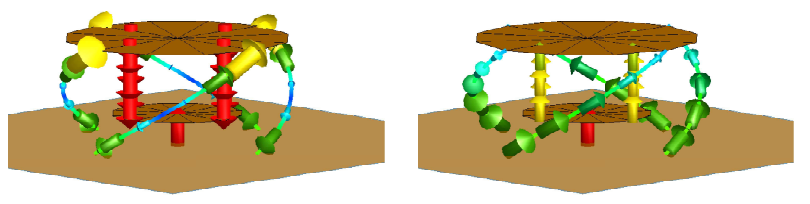

(a) $z_{2}$ mode $1.128 \mathrm{GHz}$, phase

(b) $z_{2}$ mode $1.128 \mathrm{GHz}$, phase $0^{\circ}$ $90^{\circ}$

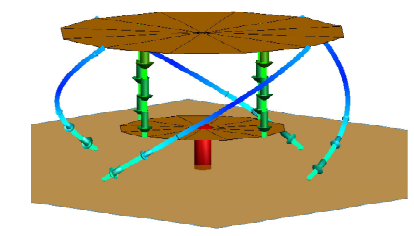

(c) Charact. mode $1.260 \mathrm{GHz}$

Fig. 17. $z_{2}$ mode and characteristic mode currents with SC input for the cage antenna.

circuited input is find at $1260 \mathrm{MHz}$. These currents are given in Fig. 17 (c).

The radiation is essentially a $T M_{01}$ mode.

5) Mode p3: $1.55 \mathrm{GHz}$ : The antenna is excited at $1.55 \mathrm{GHz}$, the wire currents are plotted in part (a) and (b) of Fig. 18.

The corresponding characteristic mode with an open-

The corresponding characteristic mode with a short- 


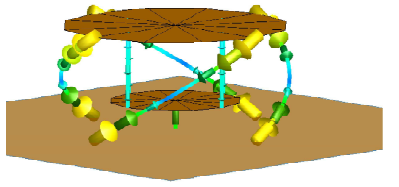

(a) $p_{3}$ mode $1.55 \mathrm{GHz}$, phase $0^{\circ}$

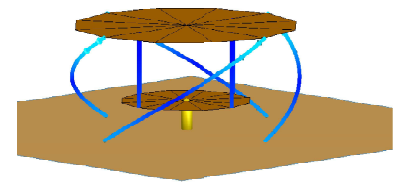

(b) $p_{3}$ mode $1.55 \mathrm{GHz}$, phase $90^{\circ}$

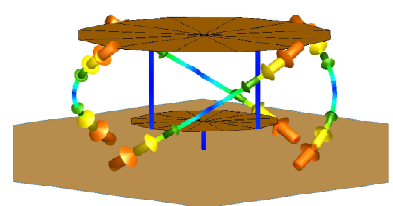

(c) Charact. mode $1.569 \mathrm{GHz}$

Fig. 18. $p_{3}$ mode and characteristic mode currents with OC input for the cage antenna.

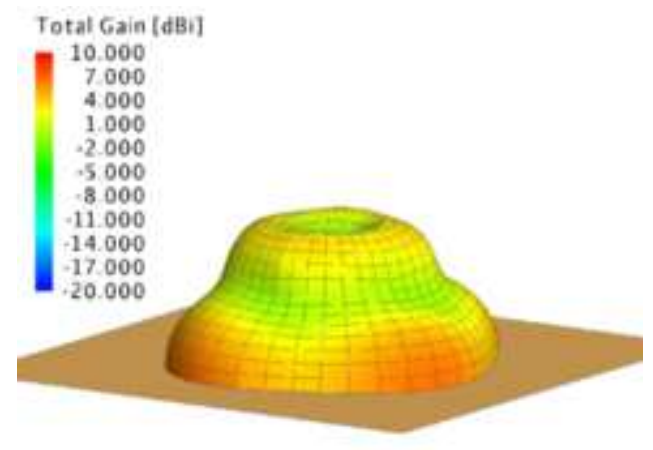

Fig. 19. Radiation pattern of the characteristic mode with an open-circuited input at $1554 \mathrm{MHz}(f c p 3)$.

circuited input is find at $1554 \mathrm{MHz}$. These currents are given in Fig. 18 (c).

The radiation is no longer a $T M_{01}$ mode and the radiation pattern is given in Fig. 19. If during the design process this mode is too close to the useful frequency band, this modal current will be strong and it will produce some pattern distortions.

\section{Antenna performances}

In Fig. 20 the gain curves versus the azimuth angles are plotted in the $\mathrm{H}$ plane for three frequencies corresponding respectively to the bottom, the center, and the top of the frequency band. We can observe an undulating gain increasing with the frequency but it remains less than $0.1 \mathrm{~dB}$ at the upper part of the bandwidth.

These undulations come from the wire currents of the helical cage as explained in [20].

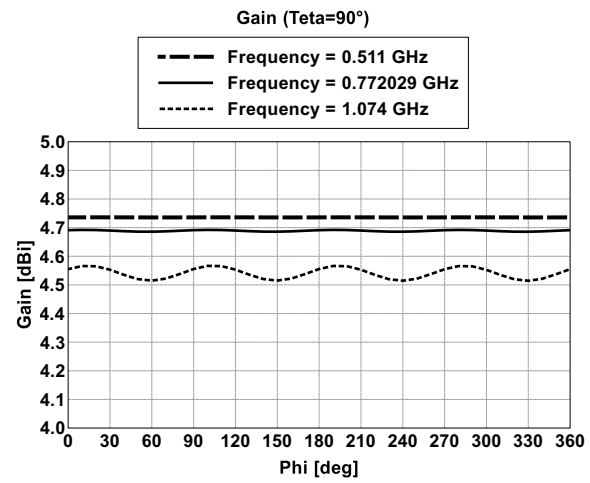

Fig. 20. Cage antenna gain (dBi) in the $\mathrm{H}$ plane ( for low, central and high operating frequency.

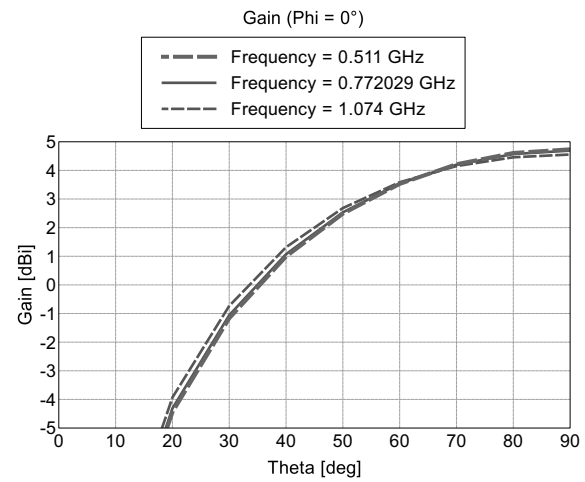

Fig. 21. Cage antenna gain (dBi) in the E plane for low, central and high operating frequency.

In Fig. 21 the gain curves versus the polar angles are plotted in the $\mathrm{E}$ plane for three frequencies corresponding respectively to the bottom, the center, and the top of the frequency band. we can verify that the gain is constant with the frequency.

The main polarization is vertical with a maximal crosspolarization at $\theta=40^{\circ}$ equal to $-25 \mathrm{~dB}$ whatever the frequency is.

In Fig. 22 the $S_{11}$ magnitude is plotted. The shape of the obtained curve is typically the one of a two order system (two pairs of conjugated poles).

\section{Meander-line and solenoid wires antennas}

The outer wires winding around the top disc presents some drawback on the polarization quality. This solution introduces a coupling between the co and the cross-polarization modes ( $T M_{01}$ and $T E_{01}$ modes). For the four wire antenna (in Sec. III-C) the cross-polarization ratio is lower than $-25 \mathrm{~dB}$

To avoid this problem, each wire is replaced by a small diameter helix (Fig. 23) or a meander-line wire (Fig. 24). In these cases, the simulation results are given in Fig. 25 where the gain is plotted in the $\mathrm{E}$ plane. The obtained cross-polarization ratio is lower than $-33 \mathrm{~dB}$ and $-47 \mathrm{~dB}$ for the helix and the meander-line wire respectively. The co-polarization gain is not affected by the kind of wires. 


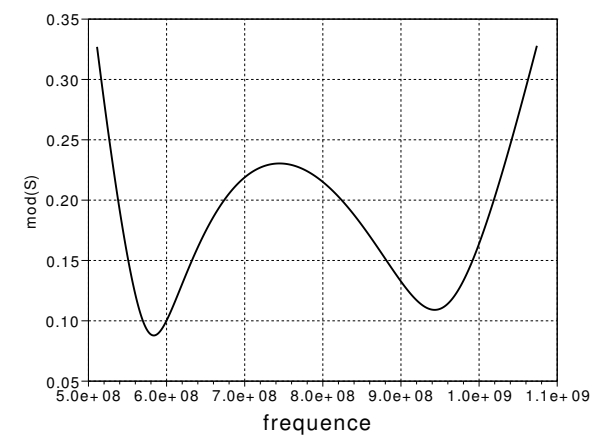

Fig. 22. Magnitude of the reflection coefficient $\left(S_{11}\right)$ of the cage antenna for a $\operatorname{VSWR}<2$ bandwidth.

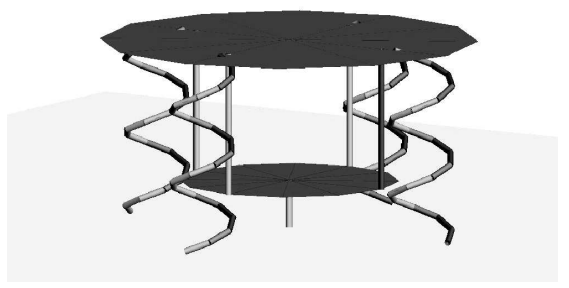

Fig. 23. Four helix wires Antenna. Dimension (see Fig.12) $h 1=6 \mathrm{~cm}$, $h 2=1.5 \mathrm{~cm}, D 1=12 \mathrm{~cm}, D 2=7 \mathrm{~cm}$, Wire diameter $2 \mathrm{~mm}$, helix diameter: $10 \mathrm{~mm}$, Nturn $=2.5$.

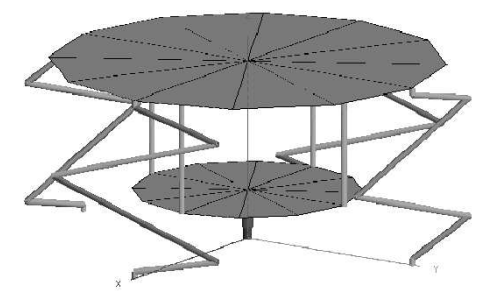

Fig. 24. Four meander lines Antenna. Dimension (see Fig.12) $h 1=6 \mathrm{~cm}$ $h 2=1.5 \mathrm{~cm}, D 1=12 \mathrm{~cm}, D 2=7 \mathrm{~cm}$, input wire diameter $1.1 \mathrm{~mm}$, All other wires diameter $1.8 \mathrm{~mm}$, meander-line width: $60 \mathrm{~cm}$.

\section{MEAsurements}

To test the antenna, a prototype has been constructed with wires and plates. The meander-line wires are chosen. Plates are $0.8 \mathrm{~mm}$ thick and the meander-line are manufactured by bending wires. Thus, a new more realistic model for simulation is now used and is shown in Fig. 26. In this model $h 1$ is the total height (including thickness of plates), the other dimensions are the same as in Fig. 12.

A picture of the prototype antenna with a small ground plane $20 \mathrm{~cm} \times 20 \mathrm{~cm}$ is given in Fig. 27. It is important to note that during measurements, a larger ground plane screwed to the small one is used $(1 \mathrm{~m} \times 1 \mathrm{~m})$.

The measured reflection coefficient is compared to the one modeled with the infinite ground plane in Fig. 28. We obtain very closed results between measurement and modeled antenna. Noise in measured data are due to parasitic reflections

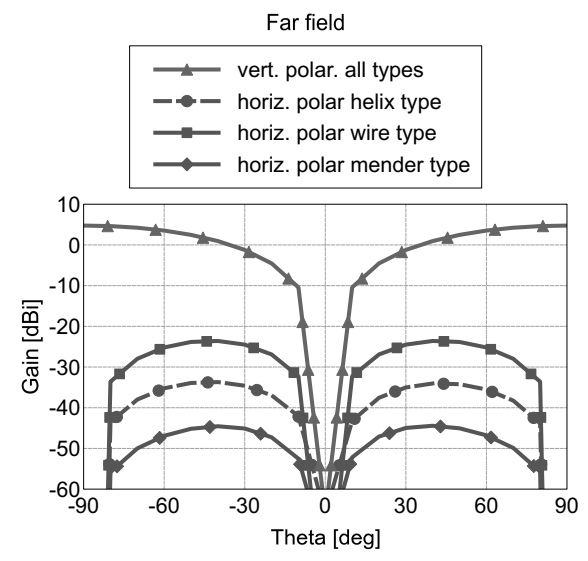

co and cross polar gain $(.75 \mathrm{GHz}, \mathrm{Phi}=0)$

Fig. 25. Co (vertical) and cross (horizontal) polarization gain (dBi) in the E plane for: Four wires antenna, four helix wires antenna, four meander lines antenna.

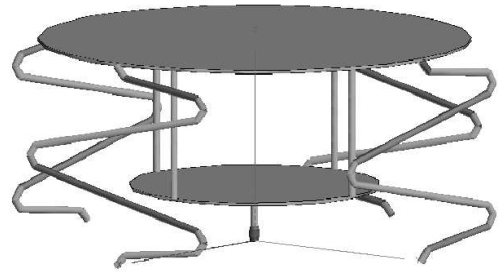

Fig. 26. Realized meander lines Antenna. Dimension (see Fig.12) $h 1=$ $6.16 \mathrm{~cm}, h 2=1.5 \mathrm{~cm}, D 1=12 \mathrm{~cm}, D 2=7 \mathrm{~cm}$, input wire diameter $1.1 \mathrm{~mm}$, All other wires diameter $1.8 \mathrm{~mm}$, meander-line width: $66 \mathrm{~cm}$, metallic plates thickness $.8 \mathrm{~mm}$.

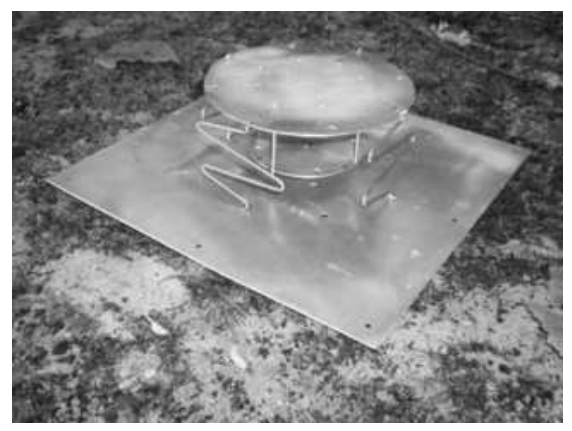

Fig. 27. Picture of the antenna with four meander-line and only a part of the ground plane (The $1 \times 1 \mathrm{~m}$ ground plane is not shown).

in the measurement environment: we don't have access to an anechoic chamber operating at these relatively low frequencies and measurements are not done in very clean environment. For this reason no pattern measurement have been performed. The obtained bandwidth at $V S W R=2$ is about $1.8: 1$ $(0.48 \mathrm{MHz} / 0.9 \mathrm{MHz})$ and $1.9: 1(0.47 \mathrm{MHz} / 0.9 \mathrm{MHz})$ for modeling and measurement respectively.

The efficiency has been estimated using simulation with a finite $1 \mathrm{~m} \times 1 \mathrm{~m}$ ground plane and all pieces made with brass. The efficiency is assessed greater than $99 \%$ over all bandwidth.

The simulated $\mathrm{H}$ plane gain pattern of the antenna on an 


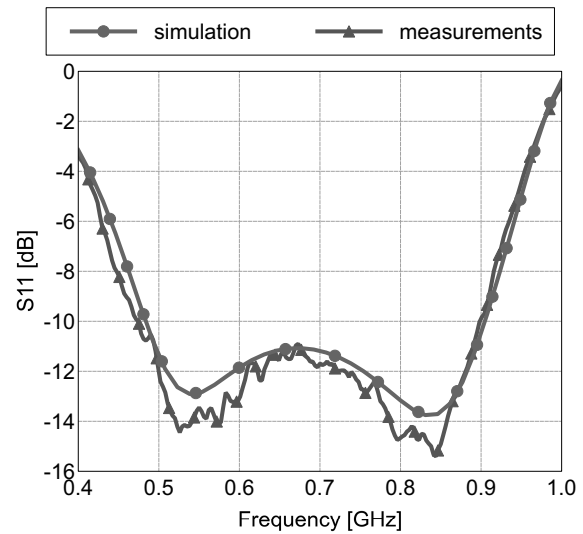

Fig. 28. Measured and Modeled reflection coefficient.

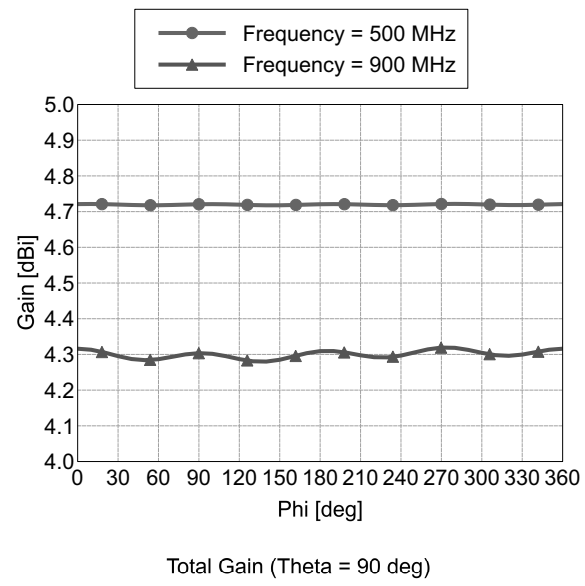

Fig. 29. Simulated meander line antenna gain in the $\mathrm{H}$ plane at lower and upper frequency in bandwidth.

infinite ground plane is given in Fig. 29 at the lower and upper frequency in the bandwidth. As we can see the radiation pattern is quite omnidirectional in the $\mathrm{H}$ plane.

\section{CONClusion}

The presented design method is based on a modal analysis using a rational fraction approximation of the input reflection coefficient. To complete this analysis it is interesting to compute characteristic modes with an open-circuited input associated with poles and characteristic modes with a short-circuited input associated with zeros. This technique allows an easy and complete understanding of the antenna operating mode, thus it is easy to identify pertinent geometric dimensions in order to obtain specific performances.

This method can be applied to every antenna structure having a finite number of (useful) poles and zeros, which is generally the case for small antennas. An antenna structure (meanderline cage antenna) having VSWR performances very similar to the ones of the Goubau antenna with similar dimensions has been designed.
Unlike Goubau antenna whose radiation pattern lack uniformity in the high part of the band the radiation pattern of the new antenna is very stable in all the bandwidth.

Manufacturing of this antenna is easy and can be done economically with plates and wires thanks to the low sensitivity to the dimension accuracy.

\section{Appendix A}

\section{A. Input impedance calculation from a current source}

The source of the Fig. 2-c is connected to a metallic structure through the two areas $\Sigma 1$ and $\Sigma 2$. The Harrington formulation [12], (Eq. 33) is used in this part.

On the $\Sigma 3$ area, the impressed current density is fixed by assumption and noticed $J s \mathbf{u}$ with $\mathbf{u}$, the unitary vector associated to the current. Then, the current source is

$$
I s=\oint_{C 1} J s d l
$$

Besides, the modal coefficient $V_{n}$ is given by

$$
V_{n}=\int_{\Sigma 3} \mathbf{E}_{\mathbf{n}} \cdot J s \mathbf{u} d \Sigma
$$

with $\mathbf{E}_{\mathbf{n}}$, the electrical field of the characteristic mode $n$ into the source volume.

For a lumped source, $\mathbf{E}_{\mathbf{n}}$ can be considered constant over इ3. Then,

$$
V_{n}=h \mathbf{E}_{\mathbf{n}} \cdot \mathbf{u} I s .
$$

Consider also the electrical field $\mathbf{E}$ defined everywhere in space and particularly over the source, it can be written as

$$
\mathbf{E}=\Sigma_{n} \frac{V_{n} \mathbf{E}_{\mathbf{n}}}{1+j \lambda_{n}}
$$

with $\lambda_{n}$, the eigenvalue associated to the characteristic mode $n$ as defined in [12] (Eq. 31).

If the characteristic mode $n$ is isolated, i.e. for $\omega \simeq \omega_{n}$ with $\lambda\left(\omega_{n}\right)=0$, if the electromagnetic fields are the ones of the characteristic mode $n$ alone, then this mode is dominant and

$$
\mathbf{E} \simeq \frac{V_{n} \mathbf{E}_{\mathbf{n}}}{1+j \lambda_{n}}
$$

In that conditions, the real function $\lambda_{n}$ of the real variable $\omega$ can be expended on

$$
\lambda_{n}(\omega) \simeq \lambda_{n}^{\prime}\left(\omega_{n}\right)\left(\omega-\omega_{n}\right) .
$$

Also, $\mathbf{E}$ can be considered as a function of $j \omega$ such as

$$
\mathbf{E}(j \omega) \simeq \frac{V_{n} \mathbf{E}_{\mathbf{n}}}{1+j \lambda_{n}^{\prime}\left(\omega_{n}\right)\left(\omega-\omega_{n}\right)} .
$$

Now, inserting the function of the complex variable $p$ defined as

$$
\begin{aligned}
p & =j \omega \quad \omega \in[-\infty+\infty] \\
p_{n} & =j \omega_{n}-\frac{1}{\lambda_{n}^{\prime}}
\end{aligned}
$$

and substituting (9) into (13), this equation becomes

$$
\mathbf{E}(\mathbf{p})=\frac{h \mathbf{E}_{\mathbf{n}} I s}{\lambda_{n}^{\prime}} \frac{1}{p-p_{n}} E_{n} .
$$


Over the $p$ domain defined by (14), $\mathbf{E}(p)=\mathbf{E}(j \omega)$. Then, $\mathbf{E}(p)$ is an analytic extension of $\mathbf{E}(j \omega)$.

Besides, the voltage at the current source terminals is given by

$$
U=-h \mathbf{E}(p) \cdot \mathbf{u}
$$

and the input impedance is defined as

$$
Z_{\text {in }}=\frac{U}{I s} \text {. }
$$

Then, when substituting (15) into (16) and (16) into (17) it follows

$$
Z_{\text {in }} \simeq-\frac{\left(h \mathbf{E}_{\mathbf{n}} \cdot \mathbf{u}\right)^{2}}{\lambda_{n}^{\prime}} \frac{1}{p-p_{n}} .
$$

The relation (18) shows that if a characteristic mode with an OC input is isolated, namely that, close to the associated frequency the field in the excited structure is quite the field of this mode, it is associated to a complex pole of the input impedance $Z_{i n}(p)$ which is also a zero of the input admittance $Y_{i n}(p)$ and the zeros of the admittance correspond to the eigenmodes with a OC input [7]. Dualistically, if a characteristic mode with a SC input is isolated, then it is associated to a complex pole of the input admittance $Y_{i n}(p)$ which is also a zero of the input impedance $Z_{i n}(p)$ and the zeros of the impedance correspond to the eigenmodes with a SC input [7].

Otherwise, from the classical study of RLC circuits in the complex domain [7], the eigenmode associated to a pole $p_{n}$ of the impedance is all the more dominant so the quality factor given in 19 presents strong values for a harmonic state.

$$
\begin{aligned}
P_{n} & =\alpha_{n}+j \omega_{n} \\
Q & =\frac{\omega_{n}}{2\left|\alpha_{n}\right|} \\
& =\frac{\omega_{n}\left|\lambda_{n}^{\prime}\right|}{2}
\end{aligned}
$$

The Q value of pole given by (19) is the same as the Q value of characteristic mode given by Harrington in [26]. Then, the quality factor $Q$ is a good characteristic to identify a dominant (isolated) mode in the operation of an antenna.

\section{REFERENCES}

[1] H. A. Wheeler, "Fundamental limitations of small antennas," Proceedings of the IRE, vol. 35, no. 12, pp. 1479-1484, 1947.

[2] L. Akhoondzadeh-Asl, J. Hill, J.-J. Laurin, and M. Riel, "Novel low profile wideband monopole antenna for avionics applications," IEEE Transactions on Antennas and Propagation, vol. 61, no. 11, pp. 57665770, 2013.

[3] M. Li and N. Behdad, "A compact, capacitively fed uwb antenna with monopole-like radiation characteristics," IEEE Transactions on Antennas and Propagation, vol. 65, no. 3, pp. 1026-1037, 2017.

[4] G. Goubau, "Multi-element monopole antennas," in Proc. ECOM-ARO Workshop on Electrically Small Antennas, 1976, pp. 63-67.

[5] L. J. Chu, "Physical limitations of omni-directional antennas," Journal of applied physics, vol. 19, no. 12, pp. 1163-1175, 1948.

[6] A. D. Yaghjian and S. R. Best, "Impedance, bandwidth, and q of antennas," IEEE Transactions on Antennas and Propagation, vol. 53, no. 4, pp. 1298-1324, 2005.

[7] H. W. Bode et al., Network analysis and feedback amplifier design. Van Nostrand Company, 1945.
[8] R. M. Fano, "Theoretical limitations on the broadband matching of arbitrary impedances," Journal of the Franklin Institute, vol. 249, no. 1, pp. 57-83, 1950.

[9] H. R. Stuart, "Eigenmode analysis of small multielement spherical antennas," IEEE Transactions on Antennas and Propagation, vol. 56, no. 9, pp. 2841-2851, 2008.

[10] — "Eigenmode analysis of a two element segmented capped monopole antenna," IEEE Transactions on Antennas and Propagation, vol. 57, no. 10, pp. 2980-2988, 2009.

[11] R. Garbacz and R. Turpin, "A generalized expansion for radiated and scattered fields," IEEE Transactions on Antennas and Propagation, vol. 19 , no. 3, pp. 348-358, 1971.

[12] R. Harrington and J. Mautz, "Theory of characteristic modes for conducting bodies," IEEE Transactions on Antennas and Propagation, vol. 19 , no. 5, pp. 622-628, 1971.

[13] _ "Computation of characteristic modes for conducting bodies," IEEE Transactions on Antennas and Propagation, vol. 19, no. 5, pp. 629-639, 1971.

[14] Y. Chang and R. Harrington, "A surface formulation for characteristic modes of material bodies," IEEE Transactions on Antennas and Propagation, vol. 25, no. 6, pp. 789-795, 1977.

[15] N. Inagaki and R. Garbacz, "Eigenfunctions of composite hermitian operators with application to discrete and continuous radiating systems," IEEE Transactions on Antennas and Propagation, vol. 30, no. 4, pp. 571-575, 1982

[16] L. Cobos, H. D. Foltz, and J. S. McLean, "A modified goubau-type antenna with two octaves of impedance bandwidth," in Antennas and Propagation Society International Symposium, 2004. IEEE, vol. 3. IEEE, 2004, pp. 3051-3054.

[17] M. Cabedo-Fabres, "Systematic design of antennas using the theory of characteristic modes," $\mathrm{PhD}$ dissertation, Universitat Politècnica de València Available at https://riunet.upv.es/handle/10251/1883, 2007.

[18] J. J. Adams and J. T. Bernhard, "Broadband equivalent circuit models for antenna impedances and fields using characteristic modes," IEEE Transactions on Antennas and Propagation, vol. 61, no. 8, pp. 39853994, 2013.

[19] S. A. Schelkunoff and H. T. Friis, Antennas: theory and practice. Wiley New York, 1952, vol. 639.

[20] C. Morlaas, B. Souny, and A. Chabory, "Cage-shaped wideband antenna based on folded monopoles," in Antennas and Propagation (ISAP), 2014 International Symposium on. IEEE, 2014, pp. 355-356.

[21] B. Gustavsen and A. Semlyen, "Rational approximation of frequency domain responses by vector fitting," IEEE Trans. Power Delivery, vol. 14, no. 3, pp. 1052-1061, July 1999.

[22] B. Gustavsen, "Improving the pole relocating properties of vector fitting," IEEE Trans. Power Delivery, vol. 21, no. 3, pp. 1587-1592, July 2006 .

[23] D. Deschrijver, M. Mrozowski, T. Dhaene, and D. D. Zutter, "Macromodeling of multiport systems using a fast implementation of the vector fitting method," IEEE Microwave and Wireless Components Letters, vol. 18, no. 6, pp. 383-385, June 2008.

[24] R. Zeng and J. Sinsky, "Modified rational function modeling technique for high speed circuits," IEEE MTT-S Int. Microwave Symp. Dig. San Francisco, CA, June 11-16 2006.

[25] R. Harrington, Time-harmonic electromagnetic fields. John Wiloy\&Sons. Inc, 2001.

[26] R. Harrington and J. Mautz, "Control of radar scattering by reactive loading," IEEE Transactions on Antennas and Propagation, vol. 20, no. 4 , pp. 446-454, 1972. 